\title{
Dorsal Hippocampal Contributions to Unimodal Contextual Conditioning
}

\author{
Tim Otto and Patrick Poon \\ Program in Behavioral Neuroscience, Department of Psychology, Rutgers University, New Brunswick, New Jersey 08854
}

\begin{abstract}
Although there is general consensus that the hippocampus is not critically involved in the acquisition of fear conditioned to an explicit conditioned stimulus (CS), the extent to which the hippocampus participates in contextual fear conditioning remains unclear. To further characterize the potential role of the hippocampus in contextual fear conditioning, the present experiments examined the effect of excitotoxic lesions of dorsal hippocampus on the acquisition of a novel contextual fear conditioning paradigm in which a unimodal (olfactory) cue served to disambiguate discrete "contexts" within a single behavioral training chamber. Selective lesions of dorsal hippocampus severely attenuated olfactory contextual conditioning without affecting conditioning to an explicit auditory or olfactory CS. Additional experiments indicate that these contextual conditioning deficits cannot be attributed to a lesion-induced decrement in olfactory perception, a preferential impairment of "weak" forms of conditioning, or hyperactivity. Thus, the hippocampus appears to contribute importantly to the acquisition of fear conditioned to explicitly nonspatial, unimodal, temporally, and spatially diffuse contextual stimuli.
\end{abstract}

Key words: dorsal hippocampus; contextual conditioning; olfactory learning; associative learning; context; rat

\section{Introduction}

Among the many successful approaches used to explore the neural substrates of associative learning has been an examination of the effects of various neurobiological manipulations on the acquisition, expression, and retention of pavlovian fear conditioning in rats (Gewirtz and Davis, 2000; Otto et al., 2000; Davis, 1992; Kim and Fanselow, 1992; LeDoux, 1995). These studies have typically examined the neural bases of learned associations between a neutral conditioned stimulus (CS), such as a light, tone, or odor, and an aversive unconditioned stimulus (US), typically mild footshock. Based on the widespread and consistent finding that amygdala damage profoundly impairs the acquisition and retention of fear conditioned to both an explicit CS ("cued" fear conditioning) and to the conditioning chamber in which the US was presented ("contextual" fear conditioning), there is now a general consensus that most forms of fear conditioning depend critically on the amygdala (LeDoux, 1995) (but see Cahill et al., 1999). Although there is also general agreement that lesions of the hippocampus typically have little or no effect on cued conditioning, hippocampal contributions to contextual conditioning remain unclear. Specifically, although several early and influential studies suggested that pretraining lesions of dorsal hippocampus resulted in a severe and lasting impairment in the acquisition of contextual conditioning (Phillips and LeDoux, 1992; Kim et al., 1993; Young et al., 1994), several recent reports have found no effect (for review, see Anagnostaras et al., 2001). In an attempt to reconcile

Received Feb. 9, 2006; revised May 5, 2006; accepted May 16, 2006.

This research was supported by National Science Foundation Grants IBN0316247 and IBN9817145.

Correspondence should be addressed to Dr. Tim 0tto, Program in Behavioral Neuroscience, Department of Psychology, 152 Frelinghuysen Road, Rutgers University, Piscataway, NJ 08854. E-mail: totto@rci.rutgers.edu. DOI:10.1523/JNEUROSCI.1056-06.2006

Copyright $\odot 2006$ Society for Neuroscience $\quad$ 0270-6474/06/266603-07\$15.00/0 these discrepant findings, it has been suggested that intact contextual conditioning in animals with dorsal hippocampal damage might be subserved by conditioning to a single, unimodal cue embedded within that context (Maren et al., 1997). One purpose of the present experiments was to examine this possibility.

With few exceptions, virtually every study examining hippocampal contributions to contextual conditioning has operationally defined "context" as the specific behavioral chamber in which either the CS and US were paired or in which the US was delivered in the absence of any explicit, temporally punctate CS. Although these studies have made considerable progress in delineating a potential role of the hippocampus in contextual conditioning, examining hippocampal contributions to contextual learning in paradigms in which context is defined as the place in which training occurred is potentially confounded with the known role of the hippocampus in spatial learning (Eichenbaum et al., 1994). Thus, the development of a paradigm in which a single, unimodal cue serves to disambiguate discrete contexts will allow us to examine more fully the extent to which dorsal hippocampus contributes to the acquisition of contextual information in general, independent of its role in spatial processing. To address these issues, we recently designed a paradigm in which unimodal (olfactory) cues presented in a single behavioral chamber serve to disambiguate contexts differentially associated with tone-shock pairings. The present studies examine the effects of discrete excitotoxic lesions of dorsal hippocampus on the acquisition of fear conditioned to both this unimodal contextual cue and explicit auditory and olfactory CSs.

\section{Materials and Methods}

Subjects

Naive male Sprague Dawley rats (Harlan, Indianapolis, IN) weighing between 225 and $275 \mathrm{~g}$ at the time of surgery were used as subjects. All 
subjects were housed individually in hanging metal wire mesh cages in a colony on a $12 \mathrm{~h}$ light/dark cycle, with lights on at 7:00 A.M. All behavioral procedures occurred during the light cycle. Subjects were provided with ad libitum access to food and water. Subjects were handled for $3 \mathrm{~min}$ daily for $5 \mathrm{~d}$ before surgical procedures and behavioral training. All procedures conducted with animals have been approved by the Rutgers University Institutional Animal Care and Use Committee.

\section{Apparatus}

Fear conditioning chamber. Fear conditioning was conducted in behavioral chambers $(30 \times 24 \times 27 \mathrm{~cm}$; BRS/LVE, Beltsville, MD), each enclosed within an aluminum sound-attenuating enclosure $(56 \times 41 \times 42$ $\mathrm{cm})$. A pair of opposing walls and the ceiling of the conditioning chamber were made of transparent Plexiglas; the other pair of opposing walls was made of aluminum. The floor of the chamber consisted of 16 stainless steel rods ( $5 \mathrm{~mm}$ in diameter) spaced from $1.9 \mathrm{~cm}$ on center. These rods were connected to a shock generator (model H13-15; Coulbourn Instruments, Allentown, PA) and served to deliver the scrambled footshock US. A tray filled with sawdust was placed under the grid floor. Each chamber was equipped with a speaker and computer-operated tone generator (3.9 $\mathrm{kHz}, 70 \mathrm{~dB}$ ) mounted $14 \mathrm{~cm}$ above the floor on one of the aluminum walls. A single light bulb (28 V, $0.04 \mathrm{~A})$ was situated $10.5 \mathrm{~cm}$ above the tone speaker.

Odor was introduced into the chamber using a flow-dilution olfactometer identical in design to those used in previous studies of olfactory fear conditioning (Herzog and Otto, 1997, 1998, 1999, 2002; Otto et al., 1997; Cousens and Otto, 1998). Clean air was directed to a solenoid valve that, when operated, passed air ( 1.5 or $0.5 \mathrm{l} / \mathrm{min}$ as conditions required) to a $20 \mathrm{ml}$ bottle containing $3 \mathrm{ml}$ of either strawberry extract (McCormick, Hunt Valley, MD) or 15\% pyridine in propylene glycol. Odorized air was subsequently introduced to the conditioning chamber via $1 / 8$ inch inner diameter Tygon tubing connected to an outlet port in the ceiling. An exhaust fan mounted on the sound-attenuating enclosure continuously ventilated the inner behavioral chamber, directing the odorized air past the subject and out to a vacuum dump via $7 \mathrm{~cm}$ flexible hose.

\section{Procedure}

Surgery. Subjects were treated with atropine $(0.4 \mathrm{mg} / \mathrm{kg}$, i.p. $)$ before surgery to suppress respiratory secretion. Subjects were then anesthetized with sodium pentobarbital $(50 \mathrm{mg} / \mathrm{kg}$, i.p.). The subject's head was shaved and mounted in a stereotaxic frame (David Kopf Instruments, Tujunga, CA). The shaved area was cleaned with $20 \%$ Nolvasan solution, the scalp was incised and retracted, and six small burr holes (three per hemisphere) were drilled through the skull over the intended lesion sites. Lesions were produced by infusion of NMDA (20 $\mu \mathrm{g} / \mu \mathrm{l}$; Sigma, St. Louis, $\mathrm{MO}$ ) dissolved in $0.1 \mathrm{M}$ PBS, $\mathrm{pH}$ 7.4. NMDA was infused over 4 $\min (0.05 \mu \mathrm{l} / \mathrm{min}, 0.2 \mu \mathrm{l} / \mathrm{site})$ using a $10 \mu \mathrm{l}$ Hamilton syringe mounted in a microinjector (David Kopf Instruments), which was, in turn, attached to a standard David Kopf Instruments stereotaxic device. Infusions were made at three sites per hemisphere in the dorsal hippocampus at the following coordinates, with the dorsoventral coordinate relative to dura: [anteroposterior (AP), -2.8 ; mediolateral $(\mathrm{ML}), \pm 1.6$; dorsoventral (DV), -3.3; and AP, -4.2 ; ML, \pm 2.6 ; DV,-3.0 ; and AP, -5.3 ; ML, \pm 4.9 ; DV,-3.3$]$. After infusion at each site, the syringe was left in position for 5 min to allow for diffusion of the NMDA. On completion of all of the infusions, the incision was closed with stainless steel surgical staples and antibiotic cream was applied to the sutured area. Sham-operated subjects received the same surgical procedures, except that the tip of the syringe was lowered only to $1.5 \mathrm{~mm}$ ventral to dura. After surgery, subjects were housed in holding cages for $2 \mathrm{~d}$ for close monitoring before returning to their home cages.

Olfactory context/auditory CS conditioning. In the first study in this series, animals (lesion, $n=16$; sham, $n=16$ ) were exposed to two alternating olfactory contexts during a single $30 \mathrm{~min}$ conditioning session. Individual contexts consisted of a 5 min presentation of one odor, and each context was presented three times in an alternating manner. In the presence of one context (strawberry extract, $1.5 \mathrm{l} / \mathrm{min}$ flow rate), hereafter referred to as the "safe" context, no other explicit stimuli were presented. In the presence of the other context ( $15 \%$ pyridine, $1.5 \mathrm{l} / \mathrm{min}$ flow rate), hereafter referred to as the "unsafe" context, the animal received three presentations of a $3.9 \mathrm{kHz}$ pure tone $(20 \mathrm{~s})$, each of which coterminated with the delivery of a footshock $(2 \mathrm{~s}, 0.8 \mathrm{~mA})$. Individual tone-shock pairings were separated by a 1 min interpairing interval. Thus, each animal received nine tone-shock pairings, each of which was administered in the presence of the background olfactory unsafe context.

Twenty-four hours later, animals were tested in a novel, distinct behavioral chamber for conditioned responding to each of several relevant stimuli in a single $12 \mathrm{~min}$ test session. Presentation of individual stimuli was limited to one of the $12 \mathrm{~min}$. Specifically, the unsafe olfactory context was presented during minute 2 , the safe context was presented during minute 8 , and the CS was presented during minute 12; no stimuli were presented during the intervening minutes. Freezing behavior, characterized by a crouching posture and an absence of any visible movement except that attributable to breathing (Fanselow, 1986), was recorded continuously by an experimenter blind to the subjects' condition.

Assessment of locomotor behavior. It has been suggested that previous reports of deficits in contextual conditioning after hippocampal damage could potentially be attributed to lesion-induced locomotor hyperactivity, a response in direct competition with the freezing response (McNish et al., 1997). To assess the extent to which hippocampal lesions resulted in hyperactivity, locomotor activity was measured $24 \mathrm{~h}$ after testing in a single 6 min test session in a square open-field chamber $(85 \times 85 \times 30$ $\mathrm{cm})$ made of black Plexiglas. White lines divided the chamber floor into $3614 \times 14 \mathrm{~cm}$ squares. The chamber was placed on a table $(73 \mathrm{~cm}$ high $)$ in the center of a novel experimental room different from those used for conditioning and testing. A light fixture $(60 \mathrm{~W})$ was placed $90 \mathrm{~cm}$ above the center of the open-field chamber. The subject was placed in one corner of the chamber to start the session, and the amount of locomotor activity, defined as the number of crossings of all four legs from one square to another, was recorded by an experimenter unaware of the lesion condition of subjects.

"Foreground" olfactory contextual conditioning. Note that, in the studies described above, the unsafe contextual cue was a "background" cue in that an explicit CS was always paired with a footshock in its presence. To examine the extent to which dorsal hippocampus contributes differentially to foreground versus background unimodal contextual conditioning, separate groups of animals were trained in a paradigm identical to that described above except that no tone was presented or paired with the delivery of footshock. Thus, animals (lesion, $n=8$; sham, $n=7$ ) received the same arrangement of olfactory context and footshock presentations as in the olfactory contextual conditioning paradigm described above, but no explicit auditory CS was presented. Animals were tested $24 \mathrm{~h}$ after training in a 12 min test session as described above.

Conditioning to explicit olfactory CSs varying in associative strength with the US. Animals were trained in our standard olfactory fear conditioning paradigm in which an odorant serves as an explicit CS and not a contextual cue (Herzog and Otto, 1997, 1998, 1999; Otto et al., 1997; Cousens and Otto, 1998). Three different versions of this paradigm were used, each resulting in the acquisition of odor-shock associations differing in associative strength. One set of animals ("strong" association; lesion, $n=$ 7 ; sham, $n=8)$ received nine pairings of odorant $(1.5 \mathrm{l} / \mathrm{min})$ and footshock $(0.8 \mathrm{~mA})$, a second set of animals ("intermediate" association; lesion, $n=7$; sham, $n=7$ ) received six pairings of the same odorant and footshock, and a third set of animals ("weak" association; lesion, $n=7$; sham, $n=7)$ received six pairings of the odorant at a lower flow rate $(0.5$ $1 / \mathrm{min})$ with a weaker footshock $(0.5 \mathrm{~mA})$. Twenty-four hours after training, each group was tested in a novel behavioral chamber for fear conditioned to the odor in a 6 min test session in which the odor was presented during minutes $2-6$. Freezing responses were measured continuously by an experimenter blind to the experimental condition of the animal.

Histological preparation. After the completion of all behavioral procedures, subjects were deeply anesthetized with sodium pentobarbital (100 $\mathrm{mg} / \mathrm{kg}$ ) and were perfused transcardially with $0.9 \%$ saline, followed by a $10 \%$ solution of pH-buffered Formalin. Brains were immersed in a Formalin-sucrose solution $(0.3 \mathrm{~g} / \mathrm{l})$ for $48 \mathrm{~h}$ and were subsequently sectioned at $50 \mu \mathrm{m}$. Sections were mounted on gelatin-subbed microscope slides and stained with cresyl violet. Lesion extent was evaluated with the aid of a light microscope. 
A
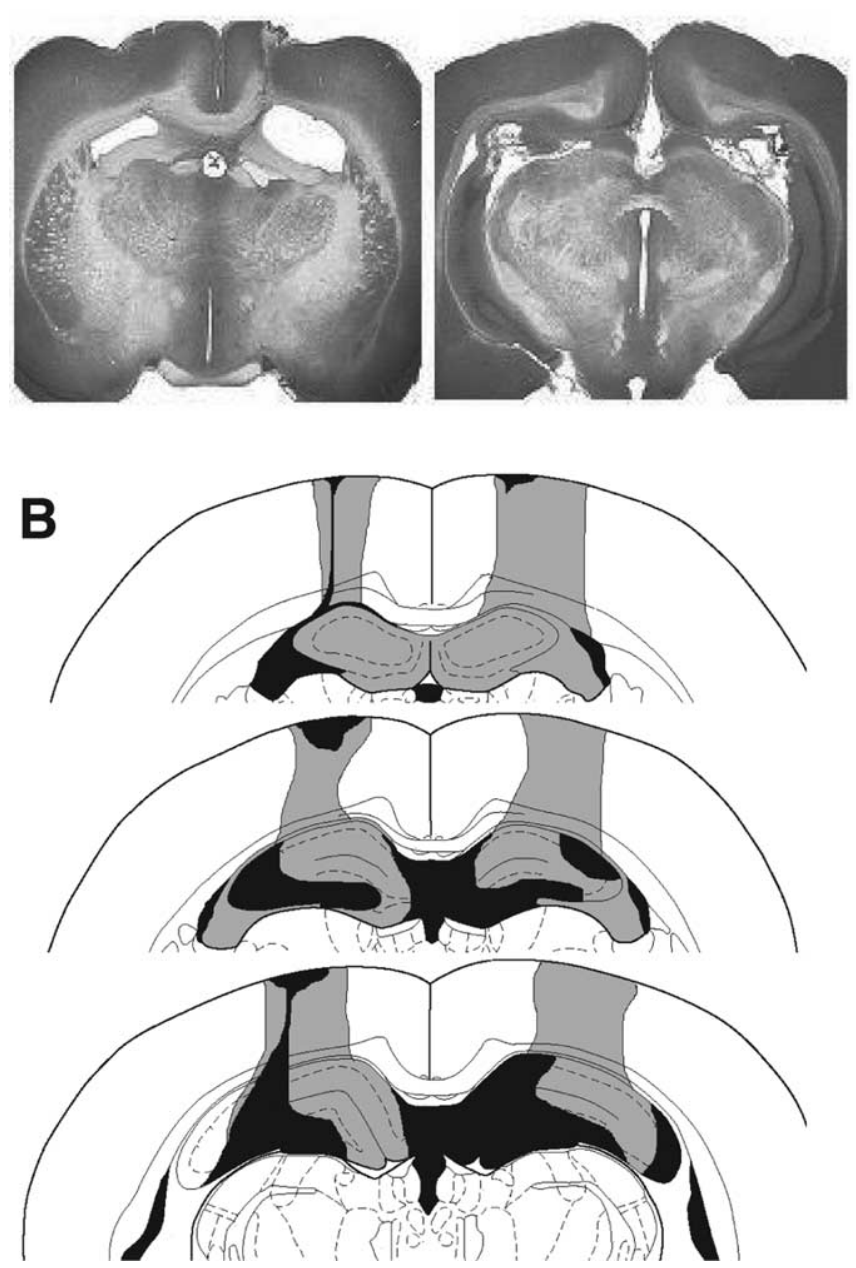

Figure 1. A, Photomicrograph of representative excitotoxic lesion of dorsal hippocampus. $\boldsymbol{B}$, Serial reconstruction of the largest (gray) and smallest (black) lesions of all animals. For additional details, see Results, Histology.

\section{Results}

\section{Histology}

The extent of damage to dorsal hippocampus for all animals is illustrated in Figure 1. All animals exhibited significant damage to the dorsal hippocampus with minimal damage to the overlying cortex. Either unilateral or bilateral damage to the fimbria anterior to approximately -3.0 (bregma) was also observed in all animals. No animals sustained damage outside these areas.

\section{Olfactory context/auditory CS conditioning: dorsal} hippocampal lesions impair olfactory contextual conditioning but not conditioning to the auditory CS Freezing behavior recorded during the 12 min testing session $24 \mathrm{~h}$ after conditioning is illustrated in Figure 2. A two-way ANOVA with one repeated measure (minute) revealed a significant main effect for lesion condition $\left(F_{(1,330)}=6.14 ; p<0.05\right)$, a significant main effect for minute $\left(F_{(11,330)}=523.4 ; p<0.0001\right)$, and a significant condition $\times$ minute interaction $\left(F_{(11,330)}=9.23 ; p<\right.$ 0.0001). Post hoc pairwise comparisons revealed that the sham and lesion animals differed only during minute 2 , that is, during presentation of the unsafe olfactory context (Student-NewmanKeuls test, $p<0.05)$. Notably, the groups did not differ in freezing behavior during presentation of the auditory CS (minute 12;

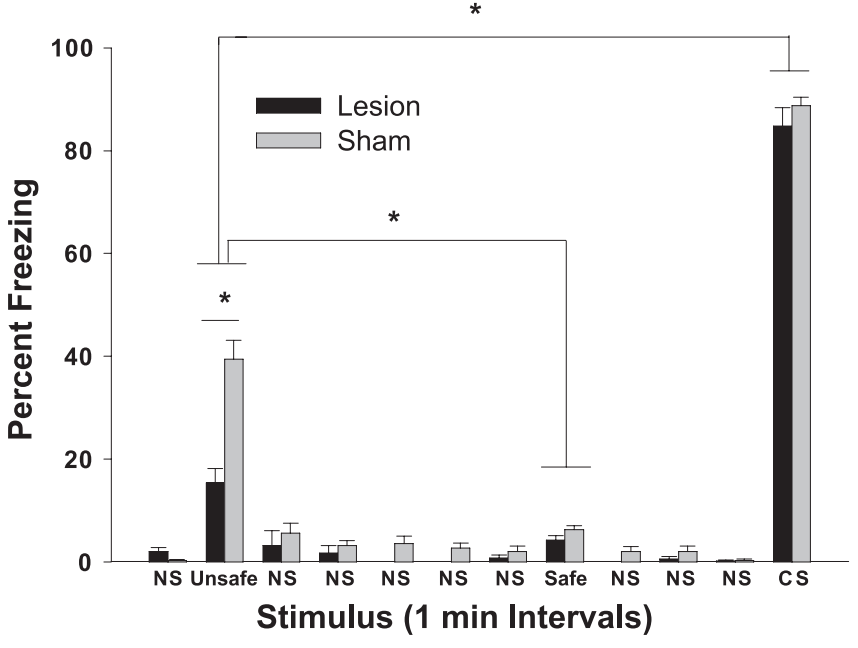

Figure 2. Freezing responses of sham-operated (Sham) animals and animals with excitotoxic lesions of dorsal hippocampus (Lesion) during the $12 \mathrm{~min}$ test session $24 \mathrm{~h}$ after olfactory context/auditory CS conditioning. Each of the relevant stimuli (unsafe context, safe context, and $\mathrm{CS}$ ) were presented for $1 \mathrm{~min}$, separated by three or more minutes of no stimulus delivery (NS). ${ }^{*} p<0.05$, Student-Newman-Keuls post hoc test.

mean sham, 88.7; mean lesion, 84.73). Finally, conditioning to the unsafe olfactory context was weaker than to the auditory CS for both groups $(p<0.05)$. Collectively, these data suggest that normal animals condition rapidly to both unimodal olfactory background contextual cues and to an auditory CS and that dorsal hippocampal lesions attenuate olfactory contextual learning without affecting conditioning to the explicit auditory CS.

Although animals with lesions of the dorsal hippocampus were impaired in conditioning to the unsafe context, they froze more during this period than during the period in which the safe context was presented $(p<0.05)$, indicating either that some of the lesioned animals learned the context-US association and others did not or that dorsal hippocampal damage does not completely eliminate context conditioning in this paradigm. To examine the extent to which lesion volume was related to the extent of impairment in conditioning to the unsafe context, the lesion size of the 16 animals with dorsal hippocampal damage was ranked by two naive observers; the inter-rater reliability as measured by Spearman's rho correlation among their rankings was $0.94(p<0.0001)$. Ranked lesion size was then correlated with ranked freezing levels recorded during presentation of the unsafe context. These analyses indicate that there is no obvious relationship between lesion size and magnitude of impairment $\left(r_{\mathrm{s}}=\right.$ 0.124 , NS). Thus, this pattern of effects cannot be attributed to an orderly relationship between lesion size and magnitude of impairment and is perhaps best described as a significant attenuation, but not a total blockade, of context-US associations in this paradigm. It should be noted that ventral hippocampus also likely contributes to contextual conditioning (Richmond et al., 1999) and that the low level of conditioning to the unsafe contextual stimulus observed in our lesioned animals may be mediated by this area.

\section{Dorsal hippocampal lesions do not result in locomotor hyperactivity}

Locomotor activity was assessed in a single 6 min test session in an open field $24 \mathrm{~h}$ after testing. Lesion and sham animals did not differ in the total amount of locomotor activity within the session 


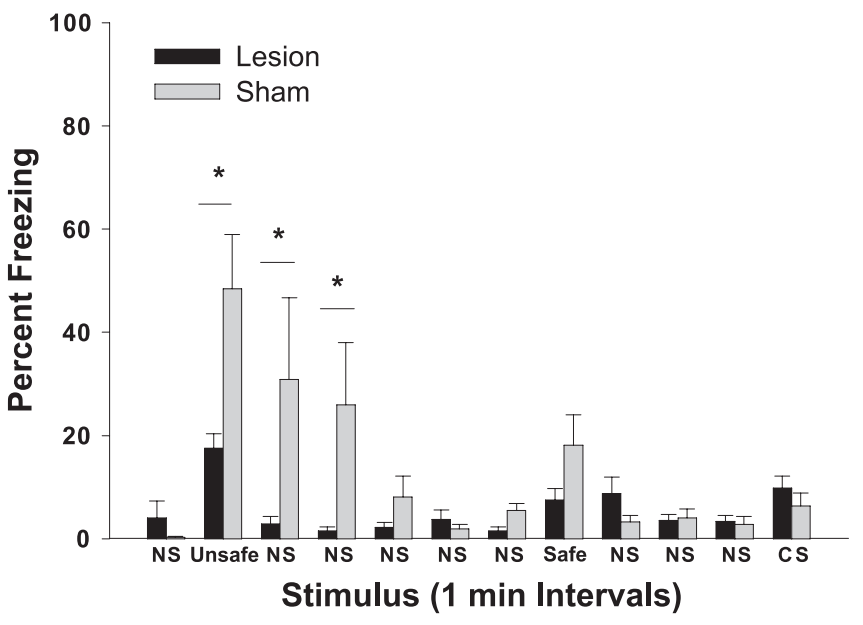

Figure 3. Freezing responses of sham-operated (Sham) animals and animals with excitotoxic lesions of dorsal hippocampus (Lesion) during the $12 \mathrm{~min}$ test session $24 \mathrm{~h}$ after olfactory foreground contextual conditioning. Each of the relevant stimuli (unsafe context, safe context, and $(S)$ were presented for $1 \mathrm{~min}$, separated by three or more minutes of no stimulus delivery (NS). Note that the tone was not presented during training the previous day. ${ }^{*} p<0.05$, Student-Newman-Keuls post hoc test.

(lesion, mean \pm SEM, $498.7 \pm 63.0$; sham, mean \pm SEM, 507.9 \pm $\left.25.4 ; t_{(30)}=-0.135, \mathrm{NS}\right)$.

\section{Dorsal hippocampal lesions impair foreground olfactory contextual conditioning}

To further explore hippocampal contributions to unimodal contextual conditioning, this experiment examined the effect of dorsal hippocampal lesions on the acquisition of foreground olfactory contextual conditioning. Specifically, training was identical to that for olfactory context/auditory CS conditioning, except that the explicit auditory CS was never presented during training. Testing was conducted $24 \mathrm{~h}$ after conditioning and was paradigmatically identical to that for background contextual conditioning described previously. Freezing behavior during the entire 12 min test session is presented in Figure 3.

A two-way ANOVA with one repeated measure (minute) revealed a significant main effect for lesion condition $\left(F_{(1,143)}=\right.$ 5.27; $p<0.05)$, a significant main effect for minute $\left(F_{(11,143)}=\right.$ 8.23; $p<0.0001)$, and a lesion condition $\times$ minute interaction $\left(F_{(11,143)}=4.46, p<.0001\right)$. Post hoc pairwise comparisons revealed a significant difference between sham and lesion conditions during minutes $2-4$, that is, during presentation of the unsafe olfactory contextual stimulus and the subsequent 2 min during which no stimuli were delivered. Note that the tone (3.9 $\mathrm{kHz}$ ), which had previously served as the explicit CS in the olfactory background contextual conditioning experiment, was presented during the final minute of testing. Because neither group exhibited significant freezing behavior during this final minute of testing, it can reasonably be concluded that the freezing responses to the CS depicted in Figure 2 cannot be attributed to unconditioned freezing behavior elicited by the tone.

For sham-operated animals, there was a trend toward elevated freezing responses during presentation of the safe olfactory context relative to other periods during which no stimuli were presented, suggesting that, perhaps in this experiment, animals were generalizing the two unimodal contexts. Post hoc comparisons revealed that freezing responses during presentation of the safe context $(18.1 \%)$ were not significantly different from those during any of the "no stimulus" epochs but were significantly differ-

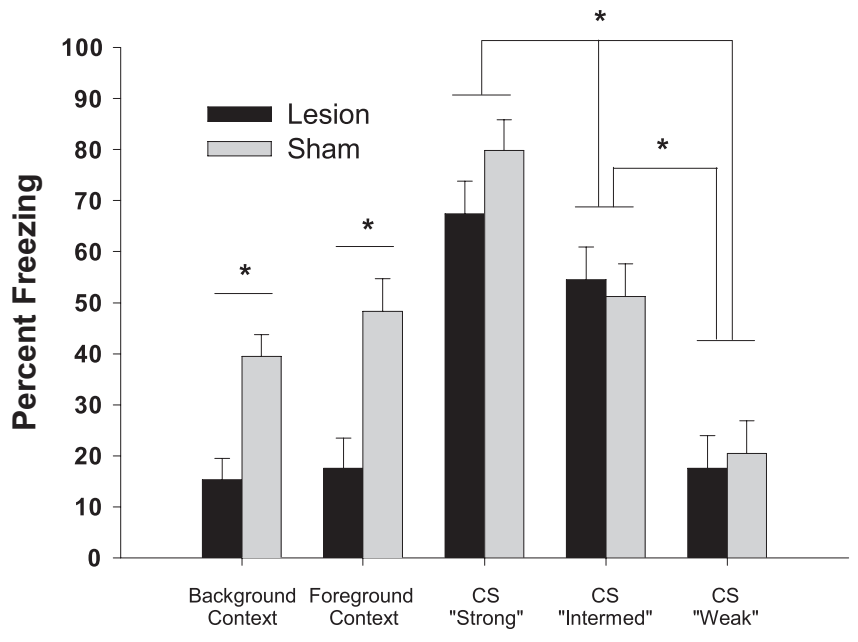

Figure 4. Left two histograms, Freezing during presentation of the unsafe background context and unsafe foreground context, replotted from Figures 2 and 3. Right three histograms, Freezing responses observed during the first minute of testing in which the odor CS was presented. CS-US pairings were modified during training to produce strong, intermediate, or weak associations. ${ }^{*} p<0.05$, Student-Newman-Keuls post hoc test.

ent from those during presentation of the unsafe context (48.4\%). Thus, from a statistical perspective, animals did not exhibit significant generalization between contexts; this is further supported by the lack of a similar trend in the background conditioning experiment illustrated in Figure 2. Nonetheless, this nonsignificant trend raises the possibility that some animals may exhibit negligible context generalization in our foreground conditioning paradigm unlike that typically found in contextual conditioning to the behavioral chamber (Kim and Fanselow, 1992).

Lesion-induced deficits in unimodal contextual conditioning cannot be attributed to a selective effect of lesions on weak associations or an inability to condition to olfactory stimuli Collectively, the results described above suggest that the hippocampus participates critically in the association of a unimodal, temporally, and spatially diffuse contextual stimulus and a footshock US. However, one alternate possibility is that dorsal hippocampal lesions may simply impair animals' ability to perceive the olfactory stimuli or to condition to them when they serve as an explicit CS. Another possibility is that, because the contextual stimuli clearly evoked weaker conditioned responses than did the explicit CS, the attenuated conditioning to the unsafe contextual stimulus may reflect differential hippocampal participation in weak versus strong associations (cf. McNish et al., 1997). An additional experiment addressed these two possibilities.

To examine whether dorsal hippocampus contributes differentially to weak versus strong CS-US associations, animals were trained in three versions of our olfactory fear conditioning paradigm (see Materials and Methods). Twenty-four hours after conditioning to explicit olfactory CSs varying in associative strength with the US, freezing responses elicited by the olfactory CS were examined. These data, together with the freezing data extracted from the foreground and background contextual conditioning experiments (unsafe context, minute 2 from Figs. 2, 3) are presented in Figure 4. A two-way ANOVA revealed a significant main effect for lesion condition $\left(F_{(1,80)}=12.75 ; p<0.001\right)$, a significant main effect for conditioning treatment $\left(F_{(4,80)}=27.1\right.$; $p<0.0001)$, and a significant lesion $\times$ conditioning interaction $\left(F_{(4,80)}=2.88 ; p<0.05\right)$. Post hoc comparisons revealed no differences between sham and lesion animals in any of the CS con- 
ditioning groups, suggesting that dorsal hippocampal lesions do not impair olfactory perception. Moreover, the strong, intermediate, and weak CS conditioning groups were all significantly different from one another $(p<0.05)$, suggesting that our paradigmatic manipulations resulted in CSs with significantly different associative strengths. Finally, there were no significant differences between performance in the intermediate CS condition and sham animals' performance in the background or foreground conditions. Based on these data, we conclude that the lesioninduced deficits observed in our olfactory contextual conditioning experiments cannot be attributed to an inability to perceive the olfactory stimulus, to condition to an olfactory stimulus when it serves as a CS, or to a differential attenuation of weak versus strong associations.

\section{Discussion}

The present results indicate that lesions of dorsal hippocampus have no effect on conditioning to an explicit auditory or olfactory CS but dramatically attenuate the acquisition of both background and foreground olfactory contextual conditioning. This deficit cannot be attributed to lesion-induced hyperactivity, an impairment of olfactory perception, an inability to condition to olfactory stimuli when they serve as phasic, discrete CSs, or the selective impairment of weak associations. Finally, note that the only paradigmatic difference between the conditions in which the odor served as a discrete, temporally punctate CS (Fig. 4, right three histograms) and those in which the odor served as a temporally diffuse, static, nonpunctate contextual stimulus (Fig. 4, left two histograms) is in the nature of the temporal relationship between that odor and the US. Thus, these data support the notion that the hippocampus is critically involved in associating a temporally and spatially diffuse, relatively nonpredictive contextual stimulus with a US.

The results of a number of previous studies indicate that pretraining electrolytic or excitotoxic lesions of dorsal hippocampus profoundly impair the acquisition of contextual fear conditioning when the context is defined as the behavioral chamber in which the US was presented (Phillips and LeDoux, 1992; Kim et al., 1993; Young et al., 1994; Maren and Fanselow, 1997). These data, which are mostly compatible with the effects of hippocampal lesions on contextual conditioning in appetitively motivated tasks (Honey and Good, 1993; Good and Bannerman, 1997) (but see Hall et al., 1996), have been viewed as consistent with the well established role of the hippocampus in "spatial" memory. The proposition that dorsal hippocampus participates critically in the acquisition of fear conditioned to spatial contextual stimuli, however, has been recently called into question for several reasons. First, several studies have reported no effect of electrolytic lesions on some forms of contextual conditioning (Phillips and LeDoux, 1994; Hall et al., 1996), whereas others report a lesioninduced enhancement of contextual learning (Winocur et al., 1987; Winocur, 1997). Second, although lesions of dorsal hippocampus have been reported to impair acquisition of contextual conditioning (Young et al., 1994, experiment 2), other studies have failed to replicate this effect (Maren et al., 1997; Richmond et al., 1999). Maren et al. (1997) have suggested that intact contextual fear conditioning after hippocampal damage may be subserved by conditioning to an individual, unimodal cue embedded within the training context. The data presented here are partially inconsistent with that hypothesis, suggesting that animals with lesions of dorsal hippocampus are impaired in conditioning to a unimodal, olfactory background or foreground contextual stimulus. It is possible, however, that this effect is limited to olfactory contextual cues and that preserved spatial contextual conditioning after hippocampal damage may be subserved by conditioning to a single cue within that behavioral chamber (cf. Anagnostaras et al., 2001).

The results of this experiment appear incompatible with those of Rawlins and Tanner (1998), who found no effect of aspiration lesions of the hippocampus on conditioning to a background auditory contextual stimulus. However, there are a number of important differences between the two studies that likely contribute to the apparent discrepancy. First, Rawlins and Tanner (1998) used an extensive training paradigm in which animals received many conditioning sessions over the course of $90 \mathrm{~d}$. It is possible that this overtraining attenuated the effect of hippocampal lesions, consistent with the data of Maren (1999) who found that extensive training mitigated the deficit in fear conditioning normally observed after amygdala lesions. Other differences include the dependent measure used (freezing vs response suppression) and the use of a delay CS-US conditioning procedure (present study) versus a trace conditioning procedure (Rawlins and Tanner, 1998).

McNish et al. (1997, 2000) have reported that, although dorsal hippocampal lesions impair the contextually elicited freezing response, they have no effect on contextually elicited fearpotentiated startle. This effect was interpreted as evidence that hippocampal lesions disrupt expression of the freezing response but do not affect contextual conditioning per se. They suggest that the "competing response" of hyperactivity often observed after hippocampal lesions may differentially affect weak versus strong associations, such that weak associations are more vulnerable to disruption than are strong associations. The present data indicate that, although contextual conditioning is indeed weaker than conditioning to an explicit olfactory or auditory CS, this difference in associative strength cannot explain the pattern of deficits observed using our paradigm. Moreover, based on the lack of effect of excitotoxic lesions on locomotor activity, the contextual learning deficits described here are not likely attributable to lesion-induced hyperactivity.

One might reasonably question our use of the term "contextual learning" in the paradigms described here. Interestingly, context has historically been defined by animal learning theorists not as the place in which some set of events occurred but rather more conceptually. Specifically, contexts are typically defined as any stimulus or set of stimuli that, relative to an explicit CS, are static, background features present during training (for review, see Balsam and Tomie, 1985). One important feature of contextual stimuli is that, relative to an explicit CS that has a temporally punctate and highly predictive relationship with a US, a contextual stimulus is more temporally (and often spatially) diffuse and relatively less predictive of the US. Experimentally, contexts have been differentiated by the presence or absence of one or more unimodal stimuli within the same chamber during training (Randrich and Ross, 1985; Nakajima, 1994; Otto et al., 1997), tones of a specific pitch (Hulse et al., 1984), the background color of a response key (Thomas, 1985), the presence or absence of a water bottle within a single chamber (Archer and Sjoden, 1980), and variations in internal state resulting from drug administration (Overton, 1964) or food deprivation (Davidson and Jarrard, 1993; Hock and Bunsey, 1998). Thus, the olfactory foreground and background contextual conditioning paradigms described here fall well within the generally accepted conceptual definition of context.

Why might the hippocampus contribute to contextual but not cued fear conditioning? Although hippocampal contributions to 
contextual learning remain to be fully characterized, there is now a general consensus that some "types" of memory are critically dependent on hippocampal processing. For example, in addition to its obvious role in spatial learning, there is also considerable evidence implicating the hippocampus in the acquisition of CS-US associations in "trace" conditioning paradigms but not in "delay" conditioning paradigms (for review, see McEchron and Disterhoft, 1999). Briefly, in delay conditioning paradigms, the CS and US overlap and typically coterminate, whereas in trace conditioning paradigms the offset of the CS occurs before the onset of the US. The deficit in trace but not delay conditioning after hippocampal damage suggests that the hippocampus is not required for the association of discrete, temporally contiguous events but may be critically involved in the association of discontiguous stimuli or events.

Wallenstein et al. (1998) (see also Levy, 1996; Wallenstein and Hasselmo, 1997) provide a theoretically compelling and biologically realistic model suggesting that the ability of the hippocampus to associate temporally discontiguous or contextual stimuli is mediated by the development of temporally overlapping "context fields," characterized by activity among asymmetrically and sparsely interconnected networks of CA3 pyramidal cells. According to this model, these context fields can mediate associations between stimuli whose temporal disparity or relationship falls outside that normally required for the induction of synaptic plasticity. This notion is consistent with the effect of hippocampal lesions on trace conditioning and with the lack of effect of hippocampal lesions on delay conditioning. Unlike explicit CSs in delay conditioning, however, contextual stimuli are temporally diffuse, static stimuli sharing a relatively imprecise and less predictive relationship with the US. Thus, in the absence of continued representation of contextual information among these overlapping context fields, animals without a hippocampus are unable to associate this temporally diffuse context information with the US. By this account, the effect of hippocampal damage on both trace and contextual conditioning may reflect similar underlying neural processes. Finally, this account suggests that hippocampal damage should differentially affect learning in two paradigms differing only in the nature of the temporal relationship between stimuli. This notion is supported by the results of the present studies: dorsal hippocampal lesions had no effect on olfactory conditioning when the odor served as an explicit CS but dramatically impaired conditioning when that same odor served as either a background or foreground contextual stimulus.

Although these data suggest that the acquisition of unimodal contextual conditioning is dependent on hippocampal integrity, several questions remain unanswered. Of particular interest is a determination of why intact spatial contextual conditioning is often observed after dorsal hippocampal damage. Consistent with the suggestion of Maren et al. (1997), it is possible that, in some instances, contextual learning may be supported by associations between a single unimodal cue embedded within that context and the US and that the pattern of effects reported here are limited to olfactory contextual stimuli. More conceptually, a systematic exploration of the specific features or qualities of contextual learning that render it susceptible to hippocampal damage will ultimately be required to more fully understand hippocampal function.

\section{References}

Anagnostaras SG, Gale GD, Fanselow MS (2001) Hippocampus and contextual fear conditioning: recent controversies and advances. Hippocampus $11: 8-17$.
Archer T, Sjoden O (1980) Context-dependent taste-aversion learning with a familiar conditioning context. Physiol Psych 8:40-46.

Balsam PD, Tomie A (1985) Context and learning. Hillsdale, NJ: Erlbaum.

Cahill L, Weinberger NM, Roozendaal B, McGaugh JL (1999) Is the amygdala a locus of "conditioned fear"? Some questions and caveats. Neuron 23:227-228.

Cousens G, Otto T (1998) Both pre- and post-training lesions of the basolateral amygdala abolish the expression of olfactory and contextual fear conditioning. Behav Neurosci 112:1092-1103.

Davidson TL, Jarrard LE (1993) A role for the hippocampus is the utilization of hunger signals. Behav Neur Biol 59:167-171.

Davis M (1992) The role of the amygdala in fear and anxiety. Annu Rev Neurosci 15:353-375.

Eichenbaum H, Otto T, Cohen NJ (1994) Two functional components of the hippocampal memory system. Behav Brain Sci 17:449-517.

Fanselow MS (1986) Associative vs. topographic accounts of the immediate shock-freezing deficit in rats: implications for the response selection rules governing species-specific defensive reactions. Learn Motiv 17:16-39.

Gewirtz JC, Davis M (2000) Using Pavlovian higher-order conditioning paradigms to investigate the neural substrates of emotional learning and memory. Learn Mem 7:257-266.

Good M, Bannerman D (1997) Differential effects of ibotenic acid lesions of the hippocampus and blockade of $N$-methyl-D-aspartate receptordependent long-term potentiation on contextual processing in rats. Behav Neurosci 111:1171-1183.

Hall GH, Purves D, Bonardi C (1996) Contextual control of conditioned responding in rats with dorsal hippocampal lesions. Behav Neurosci 110:933-945.

Herzog C, Otto T (1999) Regeneration of olfactory receptor neurons following chemical lesion: time course and enhancement with growth factor administration. Brain Res 849:155-161.

Herzog CD, Otto T (1997) Odor-guided fear conditioning. II. Lesions of anterior perirhinal cortex disrupt fear conditioned to the explicit CS but not to the training context. Behav Neurosci 111:1265-1274.

Herzog CD, Otto T (1998) Contributions of anterior perirhinal cortex to olfactory and contextual fear conditioning. NeuroReport 9:1855-1859.

Herzog CD, Otto T (2002) Neuroanatomical and behavioral effects of transforming growth factor alpha administration following olfactory nerve transection. Neuroscience 113:569-580.

Hock BJ, Bunsey MD (1998) Differential effects of dorsal and ventral hippocampal lesions. J Neurosci 18:7027-7032.

Honey RC, Good M (1993) Selective hippocampal lesions abolish the contextual specificity of latent inhibition and conditioning. Behav Neurosci 107:23-33.

Hulse SH, Cynx J, Humpal J (1984) Absolute and relative discrimination in serial pitch perception by birds. J Exp Psychol Gen 13:38-54.

Kim JJ, Fanselow MS (1992) Modality-specific retrograde amnesia of fear. Science 256:675-677.

Kim JJ, Rison RA, Fanselow MS (1993) Effects of amygdala, hippocampus, and periacqueductal gray lesions on short- and long-term contextual fear. Behav Neurosci 107:1093-1098.

LeDoux JE (1995) Emotion: clues from the brain. Annu Rev Psych 46:209-235.

Levy WB (1996) A sequence predicting CA3 is a flexible associator that learns and uses context to solve hippocampal-like tasks. Hippocampus 6:579-590.

Maren S (1999) Neurotoxic basolateral amygdala lesions impair learning and memory but not the performance of conditional fear in rats. J Neurosci 19:8696-8703.

Maren S, Fanselow MS (1997) Electrolytic lesions of the fimbria/fornix, dorsal hippocampus, or entorhinal cortex produce anterograde deficits in contextual fear conditioning in rats. Neurobiol Learn Mem 67:142-149.

Maren S, Aharonov G, Fanselow MS (1997) Neurotoxic lesions of the dorsal hippocampus and Pavlovian fear conditioning in rats. Behav Brain Res 88:261-274.

McEchron MD, Disterhoft JF (1999) Hippocampal encoding of non-spatial trace conditioning. Hippocampus 9:385-396.

McNish KA, Gewirtz JC, Davis M (1997) Evidence of contextual fear after lesions of the hippocampus: a disruption of freezing but not fearpotentiated startle. J Neurosci 17:9353-9360.

McNish KA, Gewirtz JC, Davis M (2000) Disruption of contextual freezing, 
but not contextual blocking of fear-potentiated startle, after lesions of the dorsal hippocampus. Behav Neurosci 114:64-76.

Nakajima S (1994) Contextual control of Pavlovian bidirectional occasion setting. Behav Proc 32:53-66.

Otto T, Cousens G, Rajewski K (1997) Odor-guided fear conditioning. I. Acquisition, retention, and latent inhibition. Behav Neurosci 111:1257-1264.

Otto T, Cousens G, Herzog C (2000) Behavioral and neuropsychological foundations of olfactory fear conditioning. Behav Brain Res 110:119-128.

Overton DA (1964) State-dependent or "dissociated" learning produced with pentobarbital. J Comp Physiol Psych 5:3-12.

Phillips RG, LeDoux JE (1992) Differential contribution of amygdala and hippocampus to cued and contextual fear conditioning. Behav Neurosci 106:274-285.

Phillips RG, LeDoux JE (1994) Lesions of the dorsal hippocampal formation interfere with background but not foreground contextual fear conditioning. Learn Mem 1:34-44.

Randrich A, Ross RT (1985) Contextual stimuli mediate the effects of preand postexposure to the unconditioned stimulus on conditioned suppression. In: Context and learning (Balsam PD, Tomie A, eds), pp 105132. Hillsdale, NJ: Erlbaum.

Rawlins JNP, Tanner J (1998) The effects of hippocampal aspiration lesions on conditioning to the $\mathrm{CS}$ and to a background stimulus in trace conditioned suppression. Behav Brain Res 91:61-72.

Richmond MA, Yee BK, Pouzet B, Veenman L, Rawlins JNP, Feldon J, Bannerman DM (1999) Dissociating context and space within the hippocampus: effects of complete, dorsal, and ventral excitotoxic hippocampal lesions on conditioned freezing and spatial learning. Behav Neurosci 113:1189-1203.

Thomas DR (1985) Contextual stimulus control of operant responding in pigeons. In: Context and learning (Balsam PD, Tomie A, eds), pp 295321. Hillsdale, NJ: Erlbaum.

Wallenstein GV, Hasselmo ME (1997) GABAergic modulation of hippocampal population activity: sequence learning, place field development, and the phase precession effect. J Neurophysiol 78:393-408.

Wallenstein GV, Eichenbaum H, Hasselmo ME (1998) The hippocampus as an associator of discontiguous events. Trends Neurosci 21:317-323.

Winocur G (1997) Hippocampal lesions alter conditioning to conditional and contextual stimuli. Behav Brain Res 88:219-229.

Winocur G, Rawlins JNP, Gray JA (1987) The hippocampus and conditioning to contextual cues. Behav Neurosci 101:617-625.

Young SL, Bohenek DL, Fanselow MS (1994) NMDA processes mediate anterograde amnesia of contextual fear conditioning induced by hippocampal damage: immunization against amnesia by context preexposure. Behav Neurosci 108:19-29. 